Article

\title{
Characterization and In Vitro Fecal Microbiota Regulatory Activity of a Low-Molecular-Weight Exopolysaccharide Produced by Lactiplantibacillus plantarum NMGL2
}

\author{
Mengke Yao, Min Zhang, Tiantian Lai and Zhennai Yang *
}

Citation: Yao, M.; Zhang, M.; Lai, T.; Yang, Z. Characterization and In Vitro Fecal Microbiota Regulatory Activity of a Low-Molecular-Weight Exopolysaccharide Produced by Lactiplantibacillus plantarum NMGL2. Foods 2022, 11, 393. https://doi.org/ 10.3390 /foods 11030393

Academic Editor: Denis Roy

Received: 11 December 2021

Accepted: 26 January 2022

Published: 29 January 2022

Publisher's Note: MDPI stays neutral with regard to jurisdictional claims in published maps and institutional affiliations.

Copyright: (C) 2022 by the authors. Licensee MDPI, Basel, Switzerland. This article is an open access article distributed under the terms and conditions of the Creative Commons Attribution (CC BY) license (https:// creativecommons.org/licenses/by/ $4.0 /$ )
Beijing Advanced Innovation Center for Food Nutrition and Human Health, Beijing Engineering and Technology Research Center of Food Additives, Beijing Technology and Business University, No. 11 Fu-Cheng Road, Hai-Dian District, Beijing 100048, China; 1930201080@st.btbu.edu.cn (M.Y.); 1850201008@st.btbu.edu.cn (M.Z.); 1930201070@st.btbu.edu.cn (T.L.)

* Correspondence: yangzhennai@th.btbu.edu.cn; Tel.: +86-10-68984870

\begin{abstract}
The exopolysaccharide (EPS) produced by Lactiplantibacillus plantarum NMGL2 isolated from traditional fermented dairy cheese was purified chromatographically with DEAE-Sepharose and Sepharose CL-6B columns. The purified EPS was characterized by various physicochemical methods and in vitro fecal microbiota regulation assay. The results showed that the EPS had a relatively low molecular weight of $3.03 \times 10^{4} \mathrm{Da}$, and it had a relatively high degradation temperature of $245^{\circ} \mathrm{C}$ as determined by differential scanning calorimetry. Observation of the EPS by scanning electron microscopy, transmission electron microscopy, and atomic force microscopy revealed a highly branched and tangled fibrous network microstructure with many hollow microtubules and spherical particles. Structural study by 1 H NMR spectroscopy suggested that the EPS contained a tetrasaccharide repeating unit with monosaccharide components of $\beta$-galactose $(4.6 \%)$, $\alpha$-glucose $(20.6 \%)$, and $\alpha$-mannose $(74.8 \%)$. The EPS was highly resistant to hydrolysis of simulated human saliva, gastric, and intestinal juices. Moreover, the EPS beneficially affected the composition and diversity of the fecal microbiota, e.g., increasing the relative abundance of Firmicutes and inhibiting that of Proteobacteria. The results of this study indicated significant bioactivity of this novel lowmolecular-weight EPS produced by Lpb. plantarum NMGL2, which could serve as a bioactive agent for potential applications in the food and health care industry.
\end{abstract}

Keywords: Lactiplantibacillus plantarum NMGL2; exopolysaccharide; characterization; fecal microbiota

\section{Introduction}

Many species of lactic acid bacteria (LAB) can synthesize exopolysaccharides (EPSs), including capsular polysaccharides (CPS) and slime polysaccharides (SPS) [1], such as Streptococcus thermophilus, Lactobacillus acidophilus, Lacticaseibacillus rhamnosus, Lactiplantibacillus plantarum, Lacticaseibacillus casei, and Lactococcus lactis [2]. Depending on the composition of the polysaccharides, the EPSs of LAB can be classified into homopolysaccharides and heteropolysaccharides. Structurally, the repeating units of the EPSs from LAB might be linear or highly branched, with the molecular weight (Mw) of the EPSs up to about $10^{6} \mathrm{Da}$ [3]. These EPSs have been shown to possess various bioactivities, such as antitumor [4], immunomodulation, interfering with adhesion of pathogens, inhibiting proliferation of the human colon cancer cell line, and improving resistance against viral infections [5].

The functional properties of the EPSs of LAB might vary greatly with the producing microorganisms, their culture conditions, and media composition, as well as the Mw of the polysaccharides. The EPSs with high Mw had great potential in improving food structure, e.g., promoting the formation of EPS-protein network structure in the fermentation process [6], limiting the shrinkage sensitivity of fermented milk. High Mw EPSs were also shown to help microbial communities to tolerate extreme temperatures and provide better 
protection against extreme cold temperatures [7]. Meanwhile, EPSs of lower Mw had higher water solubility with relatively extended chain conformation leading to better bioactivity [8], including immunomodulatory, antioxidant, and antitumor activities [9]. The lower Mw EPSs might also be used more efficiently by intestinal microbes [10]. It was shown that bacterial EPS364 with a relatively lower Mw of $1.48 \times 10^{4} \mathrm{Da}$ was a promising antitumor drug with obvious antitumor effects by inhibiting cancer cell growth and adhesion [11].

The human intestinal microbiota is a diverse microbial community dominated by bacteria, and maintenance of the dynamic microbiota balance is of importance in the prevention and treatment of many diseases. It was reported that the EPSs produced by Lactobacillus delbrueckii ssp. bulgaricus SRFM-1 [12] and Lactococcus lactis [13] were indigestible in the human body and could reach the end of the human intestinal tract to exert their regulatory function on the intestinal microbiota. EPSs of LAB could regulate the intestinal microbiota mainly by improving the adhesion of microbes to the intestinal mucosa, providing energy for microbial growth, balancing intestinal microbiota, and improving intestinal immunity [14]. Although the potential biological functions of the EPSs from LAB were confirmed, evaluation of these activities was mainly focused on the impact on individual beneficial bacterial species (Bifidobacteria, Lactobacillus, etc.), and the impact on the whole intestinal microbiota was not well studied.

In this study, we reported a novel low-molecular-weight EPS produced by Lpb. plantarum NMGL2 that was purified chromatographically and characterized physicochemically and biologically. 16S rRNA amplification and sequencing analysis were carried out to explore the composition, abundance, and structural changes of the fecal microbiota, including beneficial and harmful bacteria under the intervention of the EPS. Meanwhile, the changes of short-chain fatty acids (SCFAs) during in vitro fermentation were determined. The present study would help researchers better understand the physicochemical properties and bioactivity of the low-molecular-weight EPS produced by Lpb. plantarum NMGL2 for potential applications in functional foods.

\section{Materials and Methods}

\subsection{Bacterial Strain and Culture Condition}

Lpb. plantarum NMGL2 was isolated from traditional fermented dairy cheese in Inner Mongolia of China and stored at $-80^{\circ} \mathrm{C}$ in Dairy Laboratory in Beijing Technology and Business University of China. Three consecutive transfers were performed at $37^{\circ} \mathrm{C}$ for $18 \mathrm{~h}$ in MRS medium (Beijing Aoboxing Co., Ltd., Beijing, China) to activate the strain from the freezing stock $\left(-80{ }^{\circ} \mathrm{C}\right)$. The skim milk medium $(10 \%)$ prepared by reconstitution of skim milk powder (Fonterra, Auckland, New Zealand) was used for the strains growth to prepare EPS samples in this study.

\subsection{Extraction and Purification of EPS}

The activated $L p b$. plantarum NMGL2 was inoculated ( $3 \% v / v)$ into liquid skim milk medium and cultured anaerobically at $37^{\circ} \mathrm{C}$ for $24 \mathrm{~h}$ to produce EPS. Then the enzymes were inactivated by heating the culture in a boiling water bath for $15 \mathrm{~min}$. The $80 \%(w / v)$ trichloroacetic acid was added to the culture, and the final concentration was adjusted to $4 \%(w / v)$, which was stirred for $2 \mathrm{~h}$ before centrifuging at $11,000 \times g$ at $4{ }^{\circ} \mathrm{C}$ for $45 \mathrm{~min}$. The supernatant without free cells and proteins was mixed with two volumes of anhydrous ethanol, stored at $4{ }^{\circ} \mathrm{C}$ for $12 \mathrm{~h}$, and centrifuged at $11,000 \times g$ at $4{ }^{\circ} \mathrm{C}$ for $30 \mathrm{~min}$. The precipitate was dialyzed and then lyophilized to obtain the crude EPS.

The crude EPS solution $(20 \mathrm{mg} / \mathrm{mL}, 5 \mathrm{~mL})$ was subjected to a DEAE-Sepharose Fast Flow anion-exchange chromatography column $(26 \times 400 \mathrm{~mm})$. During stepwise gradient elution, distilled water was used for collection of initial 1-30 tubes, $0.2 \mathrm{~mol} / \mathrm{L} \mathrm{NaCl}$ solution used for subsequent 31-70 tubes, and finally $0.5 \mathrm{~mol} / \mathrm{L} \mathrm{NaCl}$ solution used for 71-100 tubes. Each tube of $5 \mathrm{~mL}$ of eluent was collected automatically in $5 \mathrm{~min}$, and the carbohydrate content was determined by the phenol-sulfuric acid method. The eluate containing EPS 
was collected, dialyzed, lyophilized, and then further purified with a Sepharose CL-6B column $(25 \times 500 \mathrm{~mm})$ to obtain the purified EPS.

\subsection{Fourier Transform Infrared Spectroscopy Analysis}

FT-IR spectroscopy was used to identify the major functional groups of the EPS. The mixture of purified EPS $(2 \mathrm{mg})$ and $\mathrm{KBr}(200 \mathrm{mg})$ was ground to prepare the sample pellet. The FT-IR spectra were recorded in the region of $4000 \mathrm{~cm}^{-1}$ to $400 \mathrm{~cm}^{-1}$.

\subsection{Molecular Weight Determination}

The gel permeation chromatography (GPC) system including Shodex SB-806m-HQ column $(13 \mu \mathrm{m}, 300 \times 8.0 \mathrm{~mm})$ linked to an SB-G $(10 \mu \mathrm{m}, 50 \times 6.0 \mathrm{~mm})$ guard column was used to determine the molecular weight of EPS. The EPS was detected using a refractive index detector (RI) (Optilab Wyatt, Wyatt Technology, Santa Barbara, CA, USA) and a multiangle laser-light scattering detector (MALLS) (DAWN HELEOS-II Wyatt, Wyatt Technology, Santa Barbara, CA, USA). The EPS sample $(200 \mu \mathrm{L})$ was loaded to the column, which was eluted with $0.1 \mathrm{M} \mathrm{NaNO}_{3}$ solution at a flow rate of $0.5 \mathrm{~mL} / \mathrm{min}$. Data were processed with Wyatt Astra software (Version 5.3.4.14, Wyatt Technology, Santa Barbara, CA, USA).

\subsection{Thermodynamic Stability Analysis}

Differential scanning calorimetry (DSC) and thermal gravimetric analysis (TGA) were performed by a dta-dsc thermal analyzer. The EPS sample $(5 \mathrm{mg})$ was placed into an $\mathrm{Al}_{2} \mathrm{O}_{3}$ crucible. The experiments were conducted from 20 to $600{ }^{\circ} \mathrm{C}$ at a heating rate of $10^{\circ} \mathrm{C} / \mathrm{min}$. The weight loss and heat flow curve relative to temperature were used to generate the TG-DSC thermogram.

\subsection{Microstructural Analysis of EPS}

\subsubsection{Scanning Electron Microscopy Analysis}

The purified EPS sample $(5 \mathrm{mg})$ was fixed to the mica surface and coated with a gold layer on both sides. Then, the sample was observed using a scanning electron microscope (S-4800; Hitachi Ltd., Tokyo, Japan).

\subsubsection{Atomic Force Microscopy Analysis}

The purified EPS was added to distilled water and stirred at $40{ }^{\circ} \mathrm{C}$ for $2 \mathrm{~h}$ to prepare EPS solution $(1 \mathrm{mg} / \mathrm{mL})$, which was then diluted to $10 \mu \mathrm{g} / \mathrm{mL}$. A total of $5 \mu \mathrm{L}$ of diluted EPS solution was dropped on the surface and dried at $25{ }^{\circ} \mathrm{C}$. The Dimension Icon microscope (Bruker Instruments Co., Karlsruhe, Germany) was used to observe AFM images.

\subsubsection{Transmission Electron Microscopy Analysis}

A drop of EPS sample $(0.1 \mathrm{mg} / \mathrm{mL})$ was placed on a carbon copper grid for $15 \mathrm{~min}$, then stained with phosphotungstate for $3 \mathrm{~min}$ and dried completely with a vacuum desiccator. The transmission electron microscope was used to obtain images.

\subsection{Monosaccharide Composition Analysis}

To determine monosaccharide composition, the purified EPS sample $(5 \mathrm{mg}$ ) was hydrolyzed with $2 \mathrm{M}$ trifluoroacetic acid (TFA) at $120^{\circ} \mathrm{C}$ for $3 \mathrm{~h}$. After the removal of water and TFA from the sample, the methanol was added to the dry sample and evaporated under reduced pressure, and repeated five times. Then, the sample was reduced with $30 \mathrm{mg}$ of $\mathrm{NaBH}_{4}$, and $1 \mathrm{~mL}$ of acetic anhydride was added to the hydrolyzed sample for acetylation. The Agilent Technologies 7890A GC with a DB-5 capillary column $(30 \times 0.25 \mathrm{~mm})$ and flame ionization detector was used to examine the acetylated sample. The carrier gas was $\mathrm{N}_{2}$, with a flow rate of $1 \mathrm{~mL} / \mathrm{min}$. The monosaccharide composition was identified by comparing them to standard L-fucose, L-rhamnose, L-arabinose, D-galactose, D-glucose, D-xylose, D-mannose, D-fructose, and D-ribose. 


\subsection{Nuclear Magnetic Resonance Spectroscopy Analysis}

The purified EPS sample was first dissolved in $\mathrm{D}_{2} \mathrm{O}$, then freeze-dried, and this procedure was repeated twice. The sample was then redissolved in $\mathrm{D}_{2} \mathrm{O}(5 \mathrm{mg} / \mathrm{mL})$ and ready for ${ }^{1} \mathrm{H}$ NMR testing. The NMR spectrum was obtained with a Bruker AVANCE AV-600MHz spectrometer (Bruker Group, Billerica, MA, USA). Parts per million (ppm) was used to express the chemical shift.

\subsection{In Vitro Digestibility of EPS}

The saliva digestion was carried out using the previous method [15]. The EPS sample $(10.0 \mathrm{mg} / \mathrm{mL})$ was removed from a $37^{\circ} \mathrm{C}$ water bath after $2 \mathrm{~min}$ incubation, and the enzyme was inactivated by heating the incubation system in a boiling water bath. Preparation of gastric juice ( $\mathrm{pH} 1.5$ ) was performed with reference to a previous method [16]. Hydrolysis of EPS by gastric juice was performed as follows with the final concentration of $L p b$. plantarum NMGL2 EPS at $10.0 \mathrm{mg} / \mathrm{mL}$. The samples were taken out at regular intervals from a $37{ }^{\circ} \mathrm{C}$ water bath and placed in a boiling water bath for $5 \mathrm{~min}$. The simulated small intestinal juice was prepared with reference to a previous method [17]. The samples added with EPS were drawn at regular time intervals from a $37^{\circ} \mathrm{C}$ water bath, and then the enzymes were inactivated. The reducing sugar content and total content of the sample were determined by the DNS method and phenol-sulfuric acid method, respectively. The percent hydrolysis was calculated as: Hydrolysis $(\%)=[$ Reducing sugar released $/$ (Total sugar - Initial reducing sugar) $] \times 100$.

\subsection{Fecal Microbiota Regulatory Activity of EPS \\ 2.10.1. Fecal Slurry Preparation}

Preparation of fecal slurry was performed by using a previously described method with slight modifications [18]. The fresh fecal samples were provided by three healthy volunteers (ages 20-28) who had no history of gastrointestinal disease and were not treated with antibiotics within six months. The phosphate buffer solution (PBS, $1.0 \mathrm{~mol} / \mathrm{L}, \mathrm{pH} 7.0$ ) was added to the mixture of fecal samples to prepare fecal diluent in a sterile environment. The fecal diluent was homogenized for $2 \mathrm{~min}$ and centrifuged at $30 \times g$ for $5 \mathrm{~min}$ to remove the large particles. Then, the leaving liquid was used as the inoculum for the fecal fermentation.

\subsubsection{In Vitro Fecal Fermentation}

The in vitro fecal fermentation was performed by using a previous method with slight modifications [19]. Briefly, the basal culture medium was formulated as: peptone water $2 \mathrm{~g} / \mathrm{L}$, yeast extract $2 \mathrm{~g} / \mathrm{L}, \mathrm{NaCl} 0.1 \mathrm{~g} / \mathrm{L}, \mathrm{K}_{2} \mathrm{HPO}_{4} 0.04 \mathrm{~g} / \mathrm{L}, \mathrm{KH}_{2} \mathrm{PO}_{4} 0.04 \mathrm{~g} / \mathrm{L}$, $\mathrm{MgSO}_{4} \cdot 7 \mathrm{H}_{2} \mathrm{O} 0.01 \mathrm{~g} / \mathrm{L}, \mathrm{CaCl}_{2} \mathrm{H}_{2} \mathrm{O} 0.01 \mathrm{~g} / \mathrm{L}, \mathrm{NaHCO}_{3} 2 \mathrm{~g} / \mathrm{L}$, bile salts $0.5 \mathrm{~g} / \mathrm{L}$, cysteine$\mathrm{HCl} 0.5 \mathrm{~g} / \mathrm{L}$, Tween $802 \mathrm{~g} / \mathrm{L}$; pH 7.0. The medium was autoclaved $\left(121^{\circ} \mathrm{C}, 15 \mathrm{~min}\right)$, and then $1 \mathrm{~mL} / \mathrm{L}$ of hemin solution $(50 \mathrm{mg} / \mathrm{mL})$ and $10 \mathrm{~mL} / \mathrm{L}$ of vitamin $\mathrm{K}$ previously sterilized by filtration were added. The purified EPS sample $(200 \mathrm{mg})$ was added into a $10 \mathrm{~mL}$ basal culture medium and fully dissolved by oscillation to obtain a culture system with an EPS concentration of $20 \mathrm{mg} / \mathrm{mL}$. A total of $1.5 \mathrm{~mL}$ of fecal slurry was added to each culture system, and the incubation was performed inside an anaerobic cabinet (Model Plas Labs 855-AC; PLAS LABS, Inc., Lansing, MI, USA) at $37^{\circ} \mathrm{C}$. The liquid samples were taken after $4,12 \mathrm{~h}$ of fermentation. The above experimental procedure without EPS was performed simultaneously as a blank control. These experiments were repeated five times.

\subsubsection{Extraction of Genomic DNA and Amplicon Sequencing of $16 \mathrm{~S}$ rRNA Gene}

According to the previous method [20], 16S rRNA gene analysis was performed to examine the diversity of fecal microbiota. The culture system was centrifuged at $200 \times g$ for $5 \mathrm{~min}$ to remove the large particle precipitation and then centrifuged at $9000 \times g$ for 5 min to collect the bacteria. Microbial community genomic DNA was extracted using the FastDNA ${ }^{\circledR}$ (Spin Kit for Soil, MP Biomedicals, Santa Ana, CA, USA). The ABI 
GeneAmp ${ }^{\circledR} 9700$ PCR thermocycler (ABI, Foster City, CA, USA) was used to amplify the hypervariable region $\mathrm{V} 3-\mathrm{V} 4$ of the bacterial $16 \mathrm{~S}$ rRNA gene with primer pairs $338 \mathrm{~F}$ (5'-ACTCCTACGGGAGGCAGCAG-3') and 806R (5'-GGACTACHVGGGTWTCTAAT-3'). Purified amplicons were pooled in equimolar and paired-end sequenced on an Illumina MiSeq PE300 platform (Illumina, San Diego, CA, USA) according to the standard protocols by Majorbio Bio-Pharm Technology Co., Ltd. (Shanghai, China). UPARSE version 7.1 was used to cluster operational taxonomic units (OTUs) with a $97 \%$ similarity, and chimeric sequences were discovered and removed. The RDP Classifier version 2.2 was used to compare the taxonomy of each OTU representative sequence to the 16S rRNA database (e.g., Silva v138) using a confidence threshold of 0.7.

\subsubsection{Determination of Short-Chain Fatty Acids}

Short-chain fatty acids in the fecal cultures were determined by the 7890-5977 GC-MS system (Agilent Technologies Inc. Santa Clara, CA, USA). The experiment was performed according to the previous method [21] with slight modifications. Briefly, the culture was centrifuged at $9000 \times g$ for 5 min to collect the supernatant, which was added to $\mathrm{NaOH}$ $(5 \mathrm{mmol} / \mathrm{L})$ to extract SCFAs. Then one-step derivatization was carried out using propyl chloroformate in a reaction system of water, propanol, and pyridine by two-step extraction with n-hexane. The sample was injected into GC-MS, which was equipped with an HP-5 $(30 \mathrm{~m} \times 0.25 \mathrm{~mm} \times 0.25 \mu \mathrm{m})$ column using helium as the carrier gas at a flow rate of $1.0 \mathrm{~mL} / \mathrm{min}$. The oven temperature was maintained at $70^{\circ} \mathrm{C}$ for $5 \mathrm{~min}$ and then adjusted to $100{ }^{\circ} \mathrm{C}$ at the rate of $6{ }^{\circ} \mathrm{C} / \mathrm{min}$. The selection ionization mode (SIM) was selected as the detector operating mode. Six SCFA standards (Aladdin ${ }^{\circledR}$, Shanghai, China) were used for identification and quantification, including acetic acid, propionic acid, n-butyric acid, i-butyric acid, n-valeric acid, and i-valeric acid.

\subsection{Statistical Analysis}

Data were expressed as mean $\pm \mathrm{SD}$, and the statistical significance of the difference was tested by one-way ANOVA and Student's $t$-test by using SPSS 18.0 software. $p$ values $<0.05$, $0.01,0.001$ are indicated by ${ }^{*}, * * * *$, respectively.

\section{Results and Discussion}

\subsection{Extraction and Purification of EPS}

The yield of crude EPS produced by Lpb. plantarum NMGL2 was $380 \mathrm{mg} / \mathrm{L}$. The crude EPS was subjected to purification through a DEAE-Sepharose Fast Flow column to obtain one major peak of EPS and a small peak upon gradient elution (Figure 1A). Subsequent purification of the EPS by Sepharose CL-6B gel permeation chromatography (Figure 1B) showed a single peak. The purified EPS was collected for further analyses.

\subsection{Infrared Spectrum Analysis of EPS}

The FT-IR absorption spectrum of EPS was shown in Figure 2, which exhibited a variety of typical absorption peaks of polysaccharides in the range of $4000-400 \mathrm{~cm}^{-1}$. A broad and strong stretching peak at $3283.25 \mathrm{~cm}^{-1}$ indicated the presence of a significant number of hydroxyl groups $(\mathrm{O}-\mathrm{H})$, which confirmed that the compound was a polysaccharide [22]. The C-H stretching vibration led to a weak peak at $2930.81 \mathrm{~cm}^{-1}$ [23]. The absorption at $1643.36 \mathrm{~cm}^{-1}$ was due to the stretching vibration of the $\mathrm{C}=\mathrm{O}$ bond and carboxyl groups [24]. The weak stretch band at $812.26 \mathrm{~cm}^{-1}$ indicated the presence of $\alpha$-D-glucose [25]. A broad stretch of C-O-C and C-O-H at $1000-1200 \mathrm{~cm}^{-1}$, and the absorption at $1019.66 \mathrm{~cm}^{-1}$ further confirmed that the polymer was a polysaccharide [26].

\subsection{Molecular Weight Determination of EPS}

The molecular weight of the EPS from Lpb. plantarum NMGL2 was determined to be $3.03 \times 10^{4} \mathrm{Da}$ by the GPC-RID-20 system, which was similar to the EPS produced by Lpb. plantarum KX041 $\left(3.867 \times 10^{4} \mathrm{Da}\right)$ [27], but lower than the EPS from Lpb. plantarum 
DM5 [28] (1.11 × $\left.10^{6} \mathrm{Da}\right)$. Different bacterial sources, culture conditions, and hereditary characteristics may lead to differences in molecular weights.
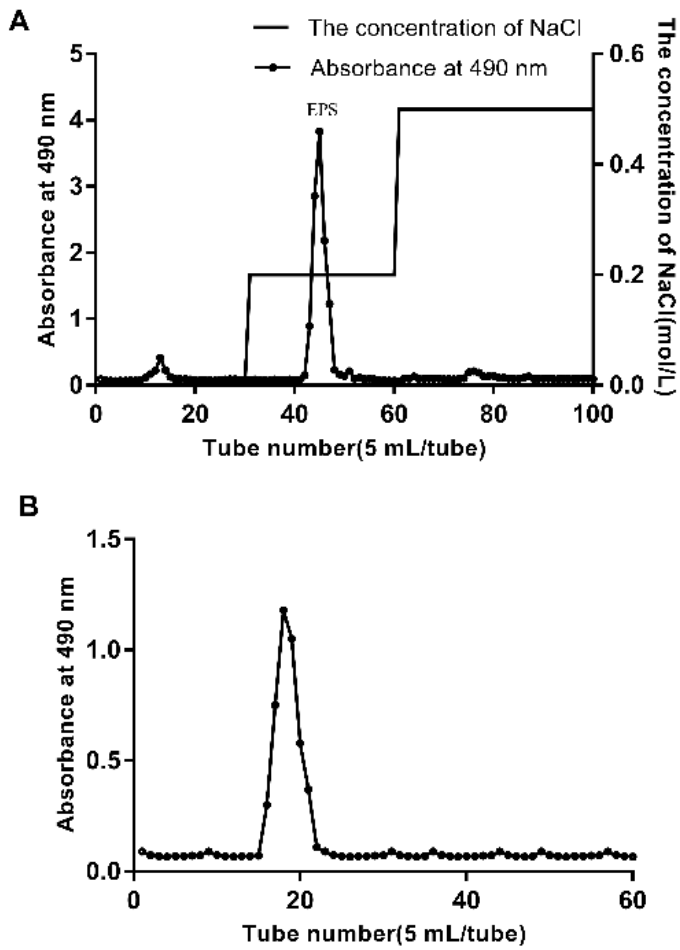

Figure 1. Purification of the crude EPS from Lpb. plantarum NMGL2 by anion-exchange chromatography on a DEAE-Sepharose Fast Flow column (A), and further gel permeation chromatography on a Sepharose CL-6B column (B).

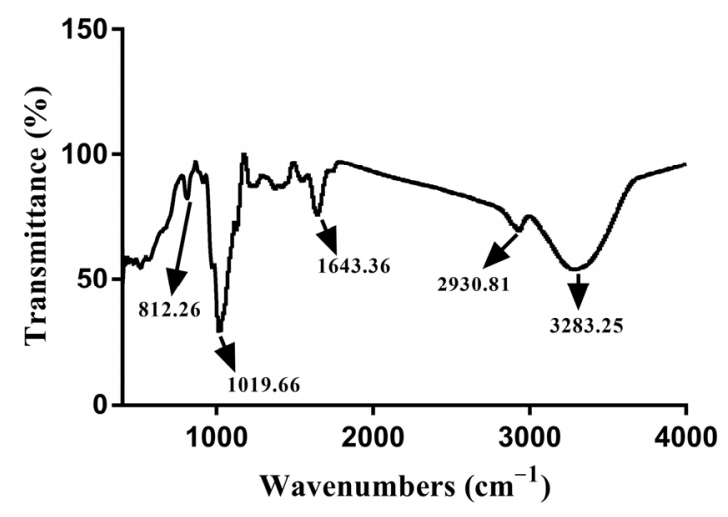

Figure 2. FT-IR spectrum of the EPS from Lpb. plantarum NMGL2 in the range of $4000-400 \mathrm{~cm}^{-1}$.

As shown in the chromatogram (Figure 3), a single and symmetrical peak was observed with the Mw/Mn ratio of the EPS of 2.71, confirming that the Lpb. plantarum NMGL2 EPS was homogeneous. The bioactivity of EPS was reported to be affected by its molecular weight, and the EPS with low molecular weight seemed to be more effective for immunomodulatory activity and antitumor activity when compared with the high molecular weight EPS [8].The EPS from Lpb. plantarum NMGL2 with a relatively lower molecular weight suggested the significance of this polysaccharide for potential application as a bioactive agent. 


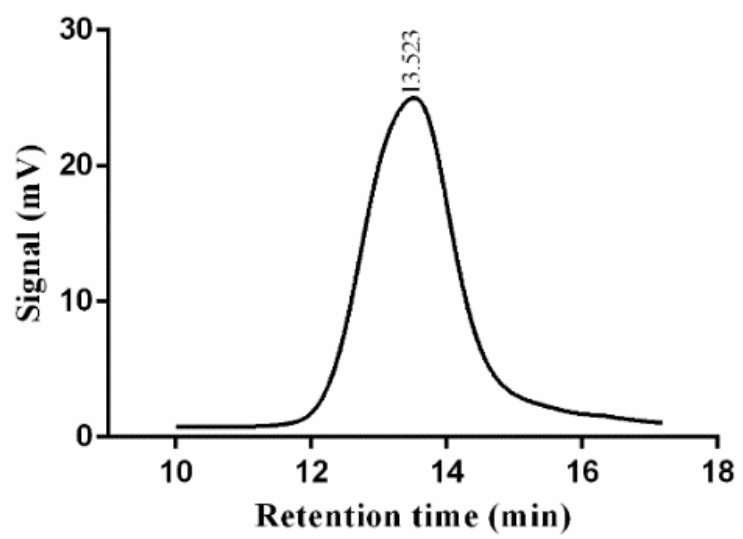

Figure 3. Molecular weight determination of the EPS from Lpb. plantarum NMGL2 by gel permeation chromatography.

\subsection{Thermogram Analysis of EPS}

TGA determines the dynamic weight loss of the purified EPS produced by Lpb. plantarum NMGL2. As shown in Figure 4, the heating process of the EPS included two steps. In the first step, an initial weight loss of approximately $10.59 \%$ was observed, which mainly attributed to the water loss in the EPS sample from 20 to $175{ }^{\circ} \mathrm{C}$. This moisture loss of mass indicated that the EPS had a high abundance of carboxyl groups, which were bound to a greater number of water molecules [29]. In the second step, there was an increased weight loss of the sample up to $50.67 \%$ at around $400{ }^{\circ} \mathrm{C}$, and about $19.08 \%$ of the total EPS was not degraded. When the EPS was heated to a high temperature, the $\mathrm{C}-\mathrm{C}$ and $\mathrm{C}-\mathrm{O}$ bonds in the ring structure were broken, leading to the release of $\mathrm{CO}, \mathrm{CO}_{2}$, and $\mathrm{H}_{2} \mathrm{O}$ [30]. The EPS started to degrade at about $245^{\circ} \mathrm{C}$, which was consistent with the fastest weight loss temperature. This indicated that the EPS had high thermal stability and had a broad application prospect in the food industry.

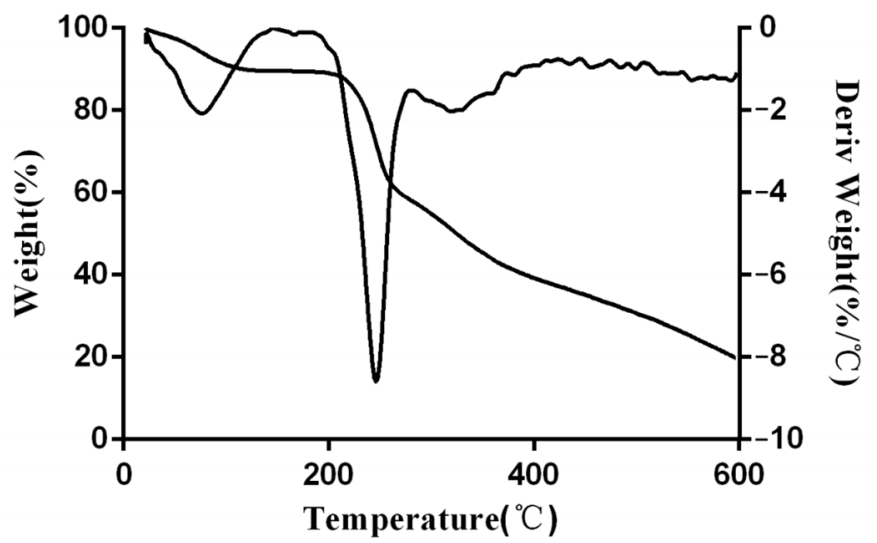

Figure 4. Thermogram curves of the EPS produced by Lpb. plantarum NMGL2.

\subsection{Microstructural Analysis of EPS \\ 3.5.1. SEM Analysis}

Scanning electron microscopy was used to study the surface morphology of macromolecules that is associated with their physical properties. As shown in Figure 5 under $1000 \times$ (Figure 5A) and 5000 $\times$ (Figure 5B) magnifications, the purified EPS produced by $L p b$. plantarum NMGL2 appeared to be a highly branched network structure with many hollow microtubules, indicating the EPS with strong water holding capacity and viscous nature that might be potentially applied in the improvement of the physical properties of foods. The EPS from L pb. fermentum Lf2 could increase the hardness and consistency index, decrease the flow behavior index of yogurt and produce yogurt with no sensory 
defects [31]. In addition, EPS could replace hydrocolloids to improve the volume, texture, and shelf life of bread [32]. A similar reticulated porous appearance was also found with the purified EPS of Lysinibacillus fusiformis KMNTT-10 [33].
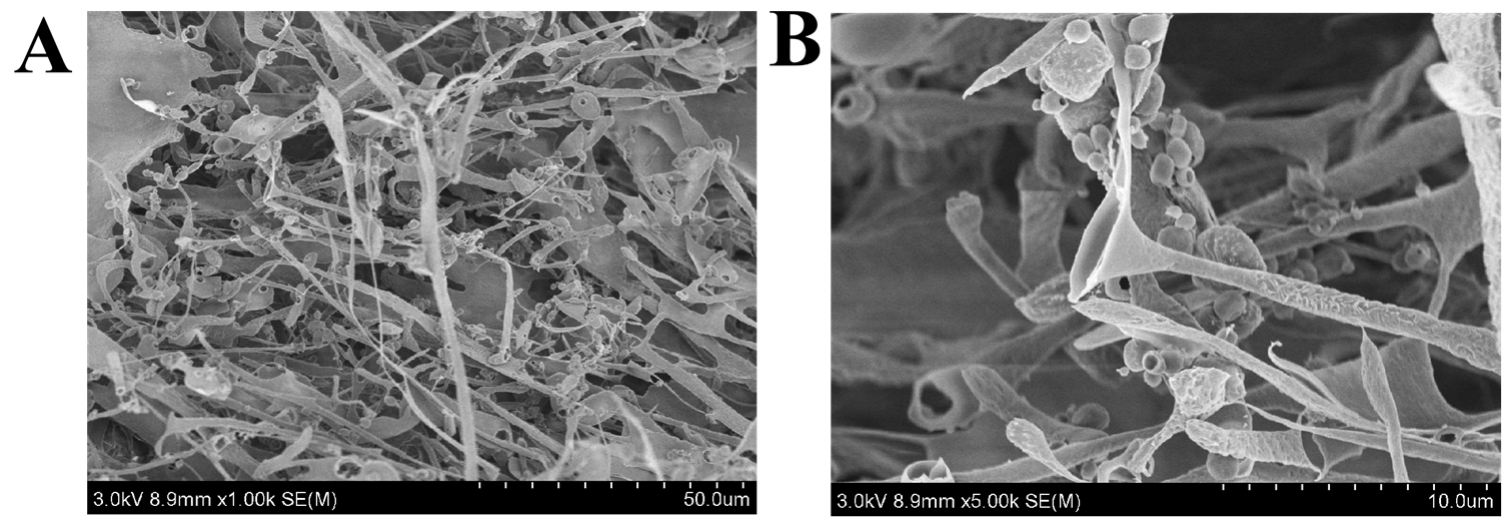

Figure 5. Scanning electron microscope images ((A): 1000×,(B): 5000×) of the EPS produced by Lpb. plantarum NMGL2.

\subsubsection{AFM Analysis}

Atomic force microscopy provides an effective approach to investigating the threedimensional microstructure of polymers. The AFM images of Lpb. plantarum NMGL2 EPS were shown in Figure 6A,B. There was the presence of many fiber structures with heights ranging from 0.4 to $7.6 \mathrm{~nm}$, which were significantly higher than those previously reported values (ca. $0.2-0.6 \mathrm{~nm}$ ) [34]. This suggested that the network structures of the EPS from $L p b$. plantarum NMGL2 might be due to the lateral association of the polymer's multimolecular chains in varying degrees. As revealed in the AFM images of the EPS (Figure 6A,B), some regions formed fibrous networks, while other regions were relatively sparse, suggesting that the EPS structure might be tangled networks. This further confirmed the complex network structure of the EPS from Lpb. plantarum NMGL2, which might lead to a polymer with higher hydrophilicity and potential to be used in food production.
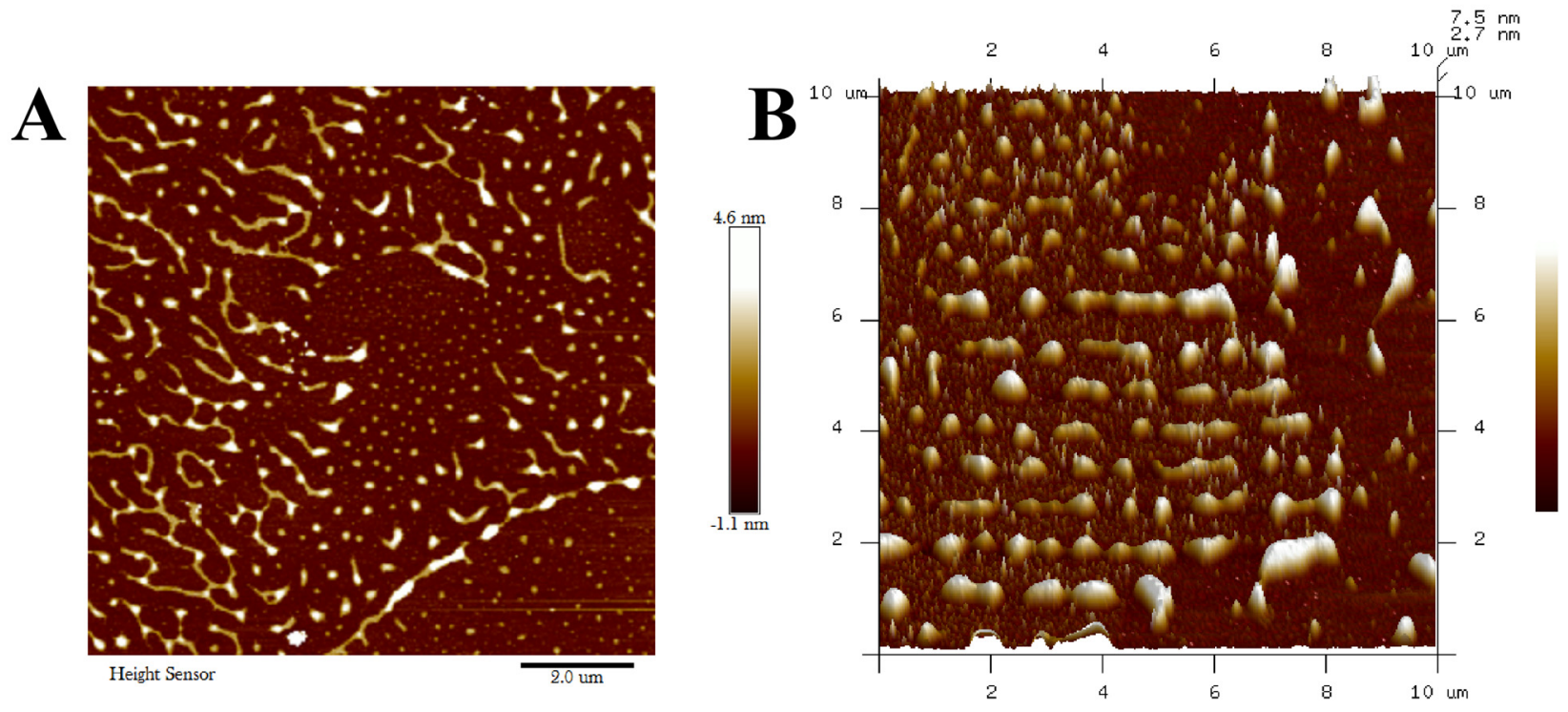

Figure 6. Atomic force microscopy images ((A): planar image; (B): cubic image) of the EPS produced by Lpb. plantarum NMGL2. 


\subsubsection{TEM Analysis}

Transmission electron microscopy is usually used to observe and analyze the ultrastructure of samples with high resolution and magnification. The TEM images of the purified EPS from Lpb. plantarum NMGL2 were presented in Figure 7A,B, demonstrating dendrimer-like microstructure that was highly branched with the presence of many spherical particles and polymer aggregates. At $1000 \times$ magnification, it could be observed that the EPS chains consisted of multiple strands of finer fibrous structures, with the smallest fibers less than $1 \mathrm{~nm}$ in diameter. The branching chains were connected with each other to form the dense structure of the polymer, which was in agreement with the observation of the EPS by SEM and AFM. Similar branched-chain morphology of the EPS from Antrodia cinnamomea as observed by TEM was reported earlier, with more flexible interchain entanglement after the addition of surfactant [35]. The microstructure of EPS was closely related to its physical properties, e.g., water binding capacity, which played an important role in maintaining the stable texture of fermented milk.
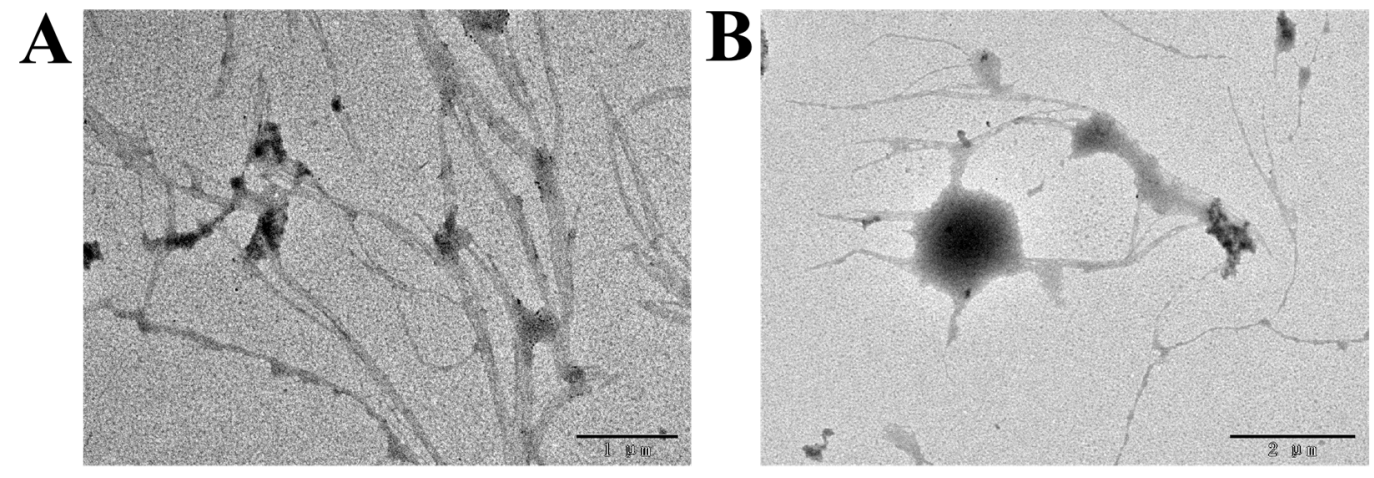

Figure 7. Transmission electron microscopic images ((A): 2000×,(B): $4000 \times)$ of the EPS produced by Lpb. plantarum NMGL2.

\subsection{Monosaccharide Composition and NMR Spectroscopy of EPS \\ 3.6.1. Monosaccharide Analysis}

Figure $8 \mathrm{~A}, \mathrm{~B}$ revealed the presence of galactose, glucose, and mannose in the EPS produced by Lpb. plantarum NMGL2, indicating that the EPS was a heteropolymer. However, the ratio of each sugar was found to be varying in percentage, such as galactose (4.6\%), glucose $(20.6 \%)$, and mannose $(74.8 \%)$. A similar kind of monosaccharide composition was observed in the EPSs of Lpb. plantarum WLPL09 [36]. However, the EPS produced by Chaetomium globosum CGMCC 6882 was composed of more sugars such as rhamnose, arabinose, galactose, glucose, xylose, mannose, galacturonic acid, and glucuronic acid [37].

\subsubsection{NMR Spectroscopy}

${ }^{1} \mathrm{H}$ NMR spectroscopy provides structural information of the monosaccharide components in the repeating unit of the bacterial EPSs by analyzing spectrum signals between 4.5 and $5.5 \mathrm{ppm}$ to distinguish the anomeric protons of sugar residues. The ${ }^{1} \mathrm{H}$ NMR spectrum of the EPS from Lpb. plantarum NMGL2 was presented in Figure 9, showing four major signals in the anomeric region at 5.22, 5.06, 5.01, and $4.97 \mathrm{ppm}$. This suggested that the EPS of Lpb. plantarum NMGL2 might be composed of a tetrasaccharide repeating unit. These resonance signals might be assigned to the anomeric protons of $\alpha$-D-glucose (5.22), $\alpha$-D-mannose (5.06), $\beta$-D-galactose (5.01), and $\alpha$-D-glucose (4.97) according to the carbohydrate research database (www.glyco.ac.ru, accessed on 25 August 2021), consisting with the results of monosaccharide analysis described above. The detailed structure of the EPS will be characterized in another study. 

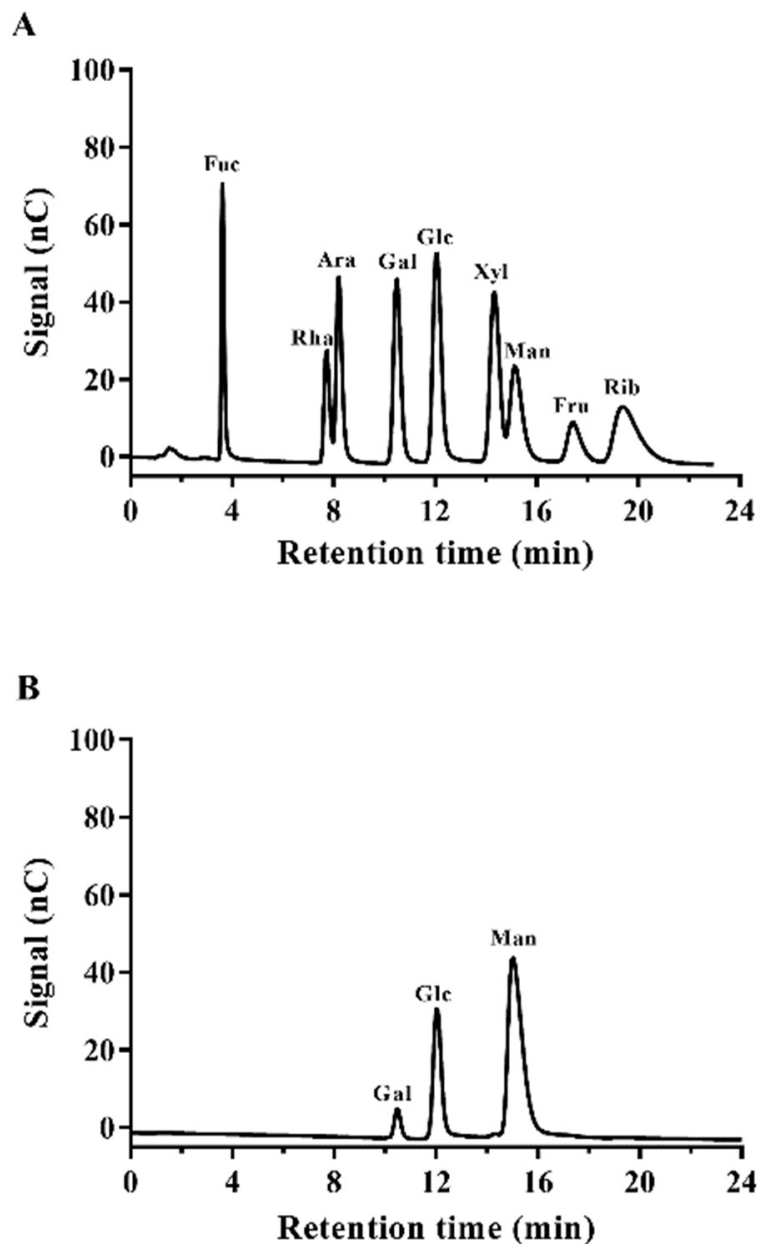

Figure 8. Gas chromatograms of the standard monosaccharides (A) and the EPS produced by $L p b$. plantarum NMGL2 (B).

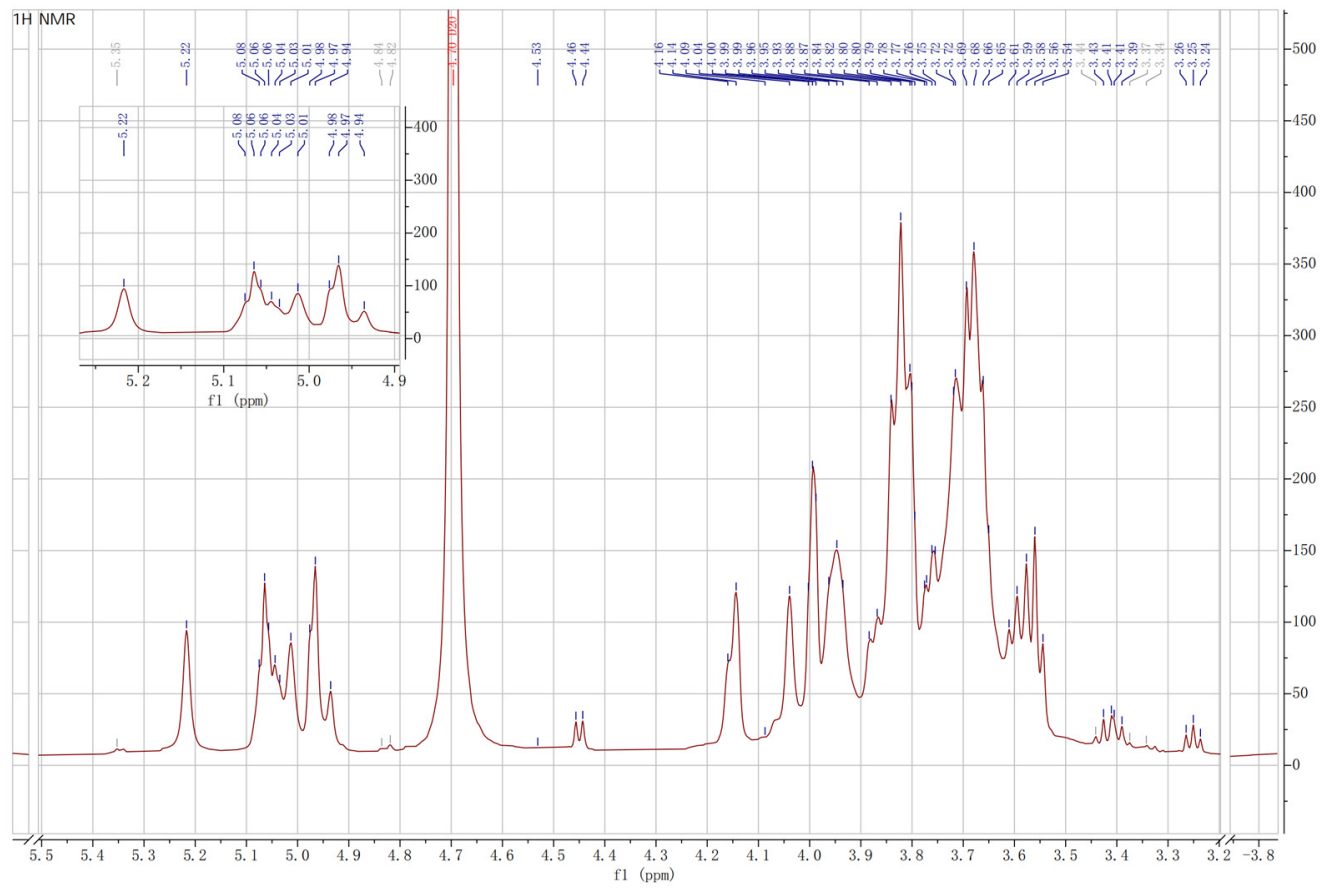

Figure 9. ${ }^{1} \mathrm{H}$ NMR spectroscopy of the EPS from Lpb. plantarum NMGL2. 


\subsection{In Vitro Digestibility of EPS}

For a substrate to be a qualified prebiotic, it should resist GIT conditions. The EPS produced from $L p b$. plantarum NMGL2 was shown with high resistance to simulated saliva, gastric juice, and intestinal juice. After $2 \mathrm{~min}$ of saliva digestion, the hydrolysis observed for the EPS was $0.54 \%$. The effect of simulated human gastric juice and intestinal juice on hydrolysis of $L p b$. plantarum NMGL2 EPS was shown in Figure 10A,B. Hydrolysis of $L p b$. plantarum NMGL2 EPS in the gastric juice increased with the incubation time. The maximum hydrolysis was $1.26 \%$ at $\mathrm{pH} 1.5$ after $6 \mathrm{~h}$. The highest hydrolysis of EPS under simulated intestinal conditions was $2.67 \%$. These results revealed that the EPS produced from $L p b$. plantarum NMGL2 could resist human digestive enzyme hydrolysis and reach the colon as a substrate for fermentation by intestinal microbiota.

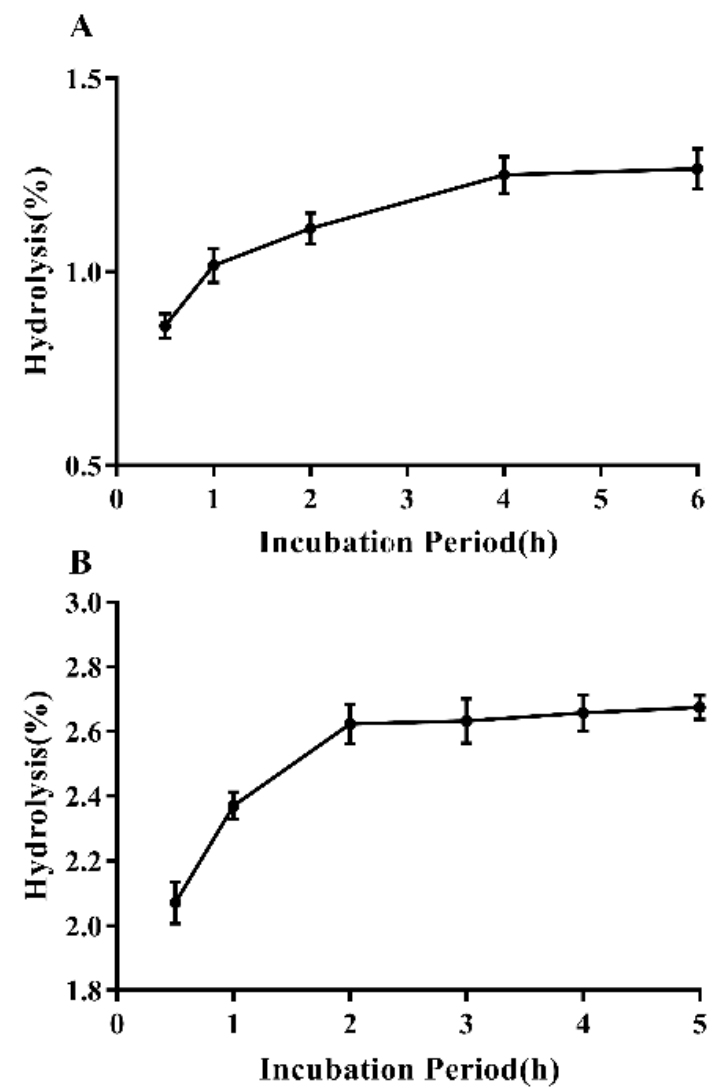

Figure 10. Hydrolysis of Lpb. plantarum NMGL2 EPS in simulated human digestive juice: (A). Gastric juice; (B). Intestinal juice.

\subsection{Fecal Microbiota Regulatory Activity of EPS}

To determine the effect of $L p b$. plantarum NMGL2 EPS on the composition of human fecal microbiota, a total of 1,264,293 sequences were obtained from 20 samples. Each sample was subsampled to the same sequencing depth (36,997 reads per sample), and clustered, 432 OTUs were obtained at $97 \%$ identity. The rarefaction curves (Figure 11A,B) for all samples reached a plateau, indicating that the sequencing depth was sufficient. The common and unique OTUs between groups were demonstrated by Venn diagram (Figure 11E). After incubation for $4 \mathrm{~h}$, the number of OTUs in the control group and EPS group were 282 and 345, respectively, and the EPS significantly increased the number of unique OTU. After incubation for $12 \mathrm{~h}$, a total of 343 and 266 OTU species were found in the control group and EPS group, respectively. Principle component analysis (PCA) revealed that different fecal coculture treatments caused unique structural changes of fecal microbiota, which was shown by clustering of samples in plots (Figure 11F). The first (PC1) and second (PC2) axes contributed $29.14 \%$ and $15.28 \%$ of the variation, respectively. 
A

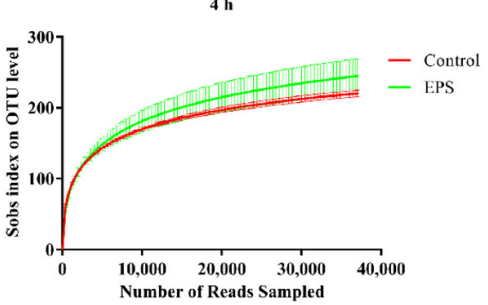

$\mathrm{B}$

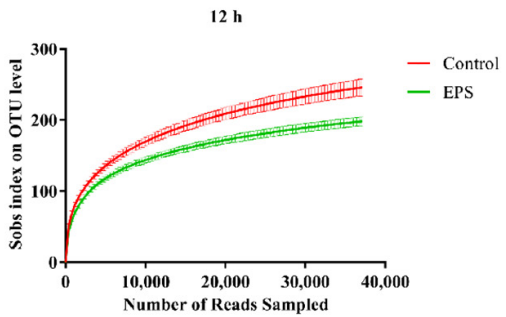

E

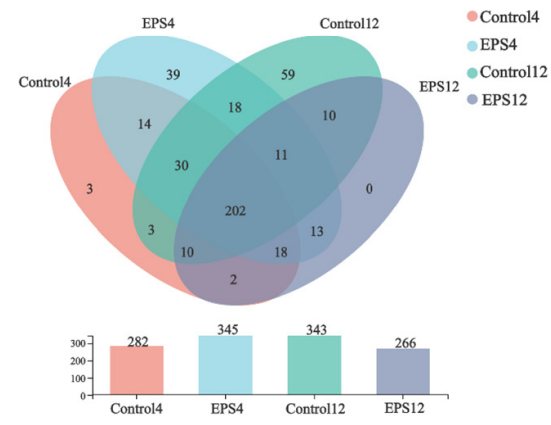

C

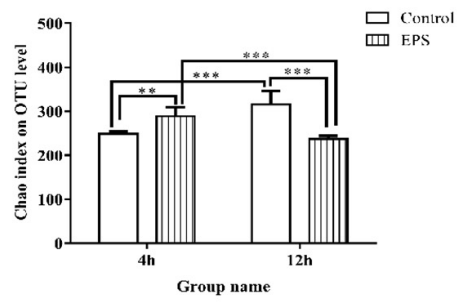

$\mathrm{D}$

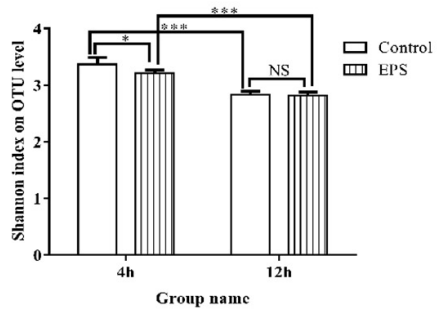

$\mathrm{F}$

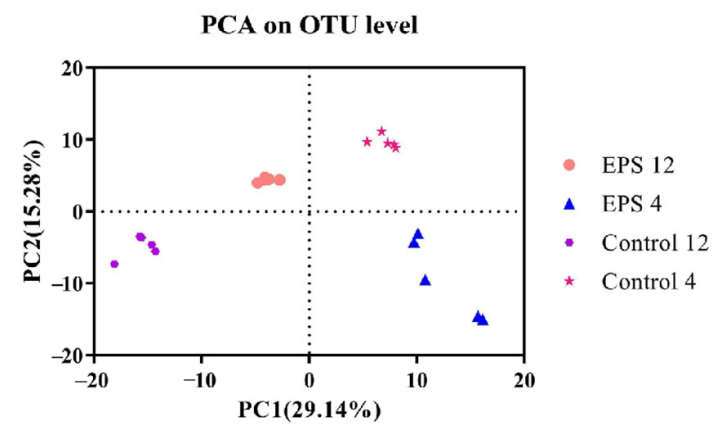

Figure 11. Analysis of DNA sequence data obtained from in vitro fecal fermentation under the intervention of the EPS produced by Lpb. plantarum NMGL2: Rarefaction curves for each experimental group with an incubation time of $4 \mathrm{~h}(\mathrm{~A})$ and $12 \mathrm{~h}(\mathbf{B}) ; \alpha$-diversity of fecal microbiota in terms of microbial richness estimates Chao1 (C) and diversity indices Shannon (D) measured at OTUs definition of $>97 \%$ identity, $p$ values $<0.05,0.01,0.001$ are indicated by ${ }^{*}, * *, * * *$, respectively; Venn diagrams showing the unique and shared OTUs of different treatment samples (E); PCA analysis of fecal microbiota samples (F).

\subsection{1. $\alpha$-Diversity Analysis}

Differences in fecal microbiota before and after EPS intervention were determined using $\alpha$-diversity (richness estimates and diversity values). Chao1 index was used to estimate richness, and Shannon index was used to estimate diversity values. After in vitro incubation for $4 \mathrm{~h}$, the Chao1 index of the EPS group was significantly higher than the control group $(p<0.01)$ (Figure 11C), but decreased Shannon index compared with the control group (Figure 11D). After incubation for $12 \mathrm{~h}$, the Chao1 index of the EPS group decreased compared with the control group, but the differences of Shannon index between the EPS group and the control group were not statistically significant.

\subsubsection{OTUs Analysis}

The differences of fecal microbiota in different groups at phylum and genus levels were analyzed (Figure 12A,B) to further evaluate the influence of EPS on fecal microbiota. At the phylum level, the fecal microbiota was composed of Firmicutes, Bacteroidetes, Actinobacteria, and Proteobacteria ( $>99.5 \%$ ) in both the samples incubated for 4 and $12 \mathrm{~h}$. After incubation for $4 \mathrm{~h}$, the relative abundance of Firmicutes increased by $42.14 \%$, and Bacteroidetes decreased by $80 \%$ in the EPS group (EPS4) compared with the control group (control4). After $12 \mathrm{~h}$ incubation of the EPS group (EPS12), the relative abundance of Actinobacteria and Bacteroidetes increased by $64.48 \%$ and $56 \%$, respectively, while Proteobac- 
teria decreased by $66.05 \%$, compared with the control group (control12). It was reported that inflammatory bowel diseases (IBD) could cause a decreased abundance of Firmicutes and increased abundance of Proteobacteria in the intestinal tract [38]. Proteobacteria was considered to be the microbial marker for dysregulation of the gut microbiota associated with many diseases [39]. The effect of the EPS produced by Lpb. plantarum NMGL2 on the fecal microbiota change could be due to the relatively low molecular weight $\left(3.03 \times 10^{4} \mathrm{Da}\right)$ and monosaccharide composition of the EPS, which benefited digestion of the polymer by the microbiota and growth of certain microbes, e.g., Firmicutes in the early fermentation stage [18]. The EPS-induced increase in the abundance of Actinobacteria containing probiotic Bifidobacterium would be beneficial to human health. Bifidobacterium played an important role in maintaining the structural integrity of intestinal mucosa, regulating the inflammatory cytokines, and preventing the passage of pathogenic bacteria and toxins [40].

A

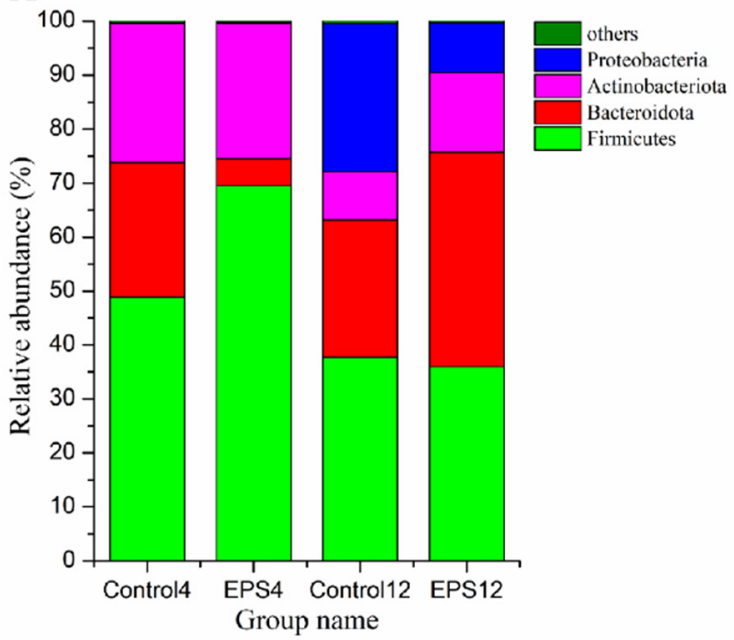

B

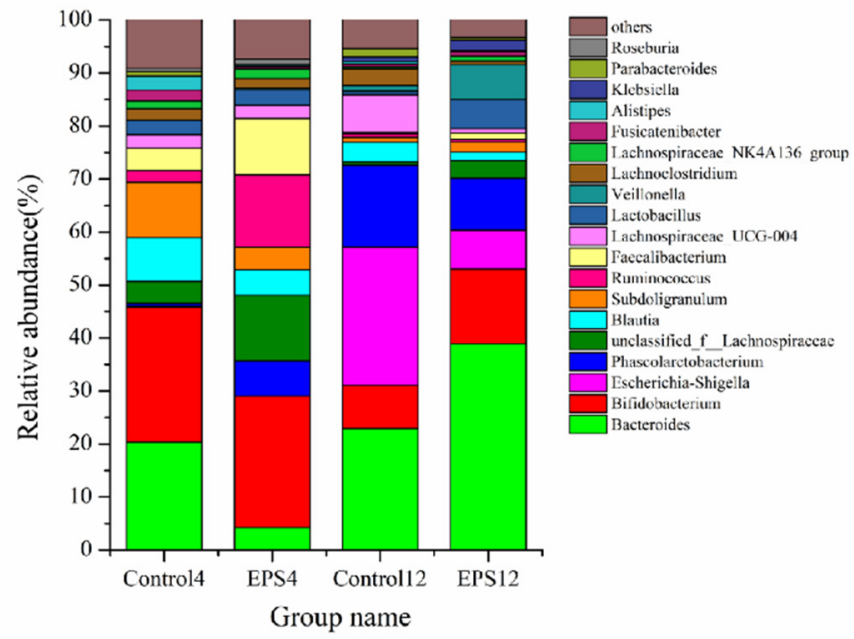

Figure 12. Comparison of fecal microbiota as affected by the EPS produced by Lpb. plantarum NMGL2 at the phylum level (A) and genus level (B) after incubation of the samples for $4 \mathrm{~h}$ (Control4 and EPS4 groups) and $12 \mathrm{~h}$ (Control12 and EPS12 groups).

At the genus level, the healthy human gut was dominant with an average relative abundance of more than 5\% of Bifidobacterium, Ruminococcus, Faecalibacterium, Phascolarctobacterium, Blautia, Subdoligranulum, Bacteroides, Lactobacillus, and unclassified_f_Lachnospiraceae. In vitro fecal fermentation with the EPS for $4 \mathrm{~h}$ the abundance of Phascolarctobacterium, unclassified_f_Lachnospiraceae, Ruminococcus, Faecalibacterium, Roseburia increased significantly $(p<0.001)$, and Bacteroides, Subdoligranulum, Fusicatenibacter, Blautia, Alistipes decreased significantly $(p<0.001)$ compared with the control group. The SCFAs were mainly produced by Firmicutes and Actinobacteria, and there was a significant increase in SCFAs producing genera in the EPS group after $4 \mathrm{~h}$ incubation, as indicated above. For instance, Phascolarctobacterium was an important producer of acetic acid and propionic acid [41], and Faecalibacterium was the main butyric acid producer that could degrade polysaccharides and had anti-inflammatory capability [42]. It was reported that propionic acid was mainly synthesized through the succinate, propanediol, and acrylate pathways [43]. There were two metabolic pathways for butyrate biosynthesis: one via butyryl-CoA/acetate CoA transferase, requiring a molecule of acetate; another one through the phosphotransbutyrylasebutyrate kinase pathway [44]. After incubation with the EPS for $12 \mathrm{~h}$, the relative abundance of Bifidobacterium $(p<0.01)$, Lactobacillus $(p<0.001)$ and Veillonella $(p<0.001)$ increased significantly, but Escherichia-Shigella, Lachnospiraceae_UCG-004, Lachnoclostridium and Parabacteroides decreased significantly $(p<0.001)$. The changes of Bifidobacterium were consistent with Actinobacteria, and Escherichia-Shigella was related to Proteobacteria. It was reported that patients with irritable bowel syndrome had significantly decreased abundance of 
intestinal Bifidobacteria. Lactobacillus could produce bacteriocins and competitively exclude potential pathogens [45].

Further linear discriminant analysis (LDA) and effective size comparisons (LEfSe) were carried out to evaluate dominant fecal microbiota (LDA; values > 4) [46] as biomarkers between groups. After incubation for $4 \mathrm{~h}$, our results showed that there were 11 and 9 significant differences in the EPS and control groups (EPS4, control4), respectively (Figure 13A). After incubation for $12 \mathrm{~h}$, it demonstrated that there were 18 and 10 significant differences in the EPS and control groups (EPS12, control12), respectively (Figure 13B). To sum up, the results described above, the EPS produced by Lpb. plantarum NMGL2 could generally increase the relative abundance of beneficial bacteria and reduce the relative abundance of harmful bacteria in the fecal microbiota.

A

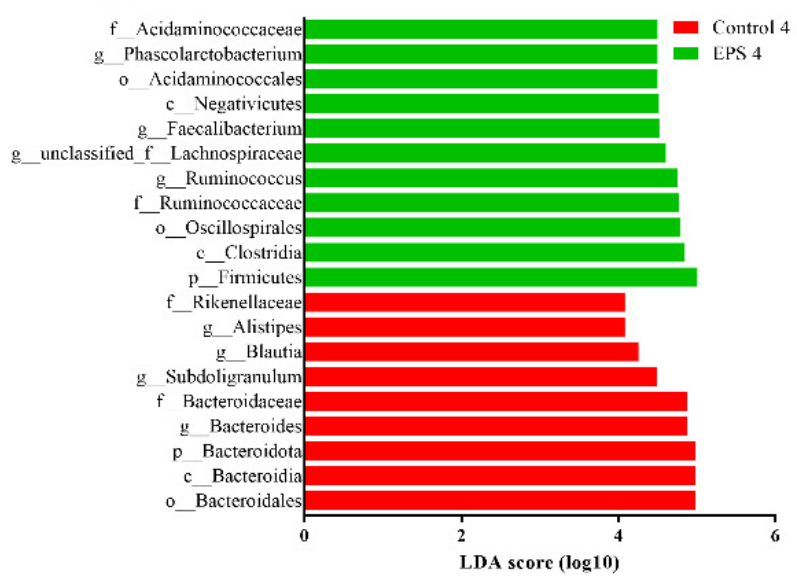

B

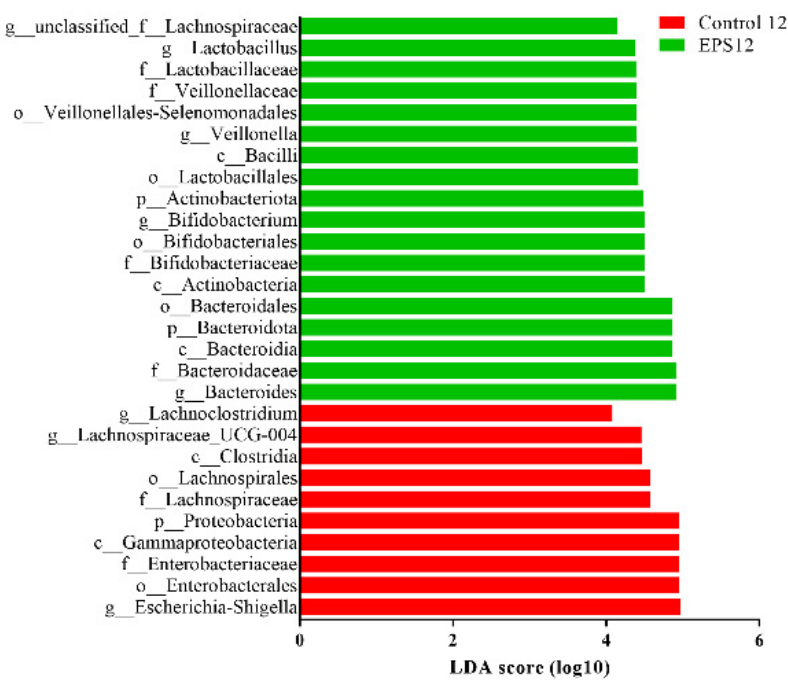

Figure 13. Comparison of fecal microbiota as affected by the EPS produced by $L p b$. plantarum NMGL2 by linear discriminant analysis (LDA score $>4$ ) generated by LEfSe analysis after incubation of the samples for $4 \mathrm{~h}$ (Control4 and EPS4 groups) (A) and $12 \mathrm{~h}$ (Control12 and EPS12 groups) (B).

\subsubsection{SCFAs Produced in the Fecal Cultures}

SCFAs include acetic, propionic, and butyric acids, which were produced by fermentation of dietary fiber and prebiotic carbohydrates by microflora. SCFAs could provide nutrition and energy for intestinal epithelial cells and play important physiological roles in the host. The increase in SCFAs is beneficial to the health of the host, such as suppressing pathogenic bacteria in the gut [47]. In addition, lactic acid produced by Firmicutes and Actinobacteria may play an important role in the regulation of Proteobacteria, especially Escherichia-Shigella. Therefore, the changes of SCFAs and lactic acid during in vitro fecal fermentation could reflect the bioactivity of $L p b$. plantarum NMGL2 EPS. Changes of SCFAs concentrations in feces during in vitro fecal fermentation as affected by the EPS produced by $L p b$. plantarum NMGL2 are shown in Figure 14. After EPS intervention for $4 \mathrm{~h}$, though there was a significant increase $(p<0.001)$ in the abundance of SCFAs producing Phascolarctobacterium and Faecalibacterium, the levels of SCFAs decreased significantly, probably due to the fact that the SCFAs were used by Firmicutes to increase their growth. Furthermore, the contents of acetic acid and propionic acid increased significantly after $12 \mathrm{~h}$. However, the concentration of the total SCFAs during the in vitro fecal fermentation was not significantly changed, probably due to the fact that the SCFAs products of some bacteria might provide substrates for others. This was also known as the cross-feeding effect that might contribute to the alteration of microbiota composition [48]. In addition, the concentration of butyrate decreased significantly compared to both control groups (4 and $12 \mathrm{~h}$ ). It was reported that SCFAs as substrates, e.g., dietary butyrate, could significantly increase the relative 
abundance of the phylum Firmicutes in mice [49]. Lactic acid was also produced during EPS fermentation in vitro, as found in other studies [12]. Therefore, the EPS may affect the fecal SCFAs and lactic acid composition, which in turn could be as substrates to regulate the fecal microbiota composition. However, the exact mechanism of the EPS-induced complex cross-feeding effect involving SCFAs on fecal microbiota changes needs to be further studied.
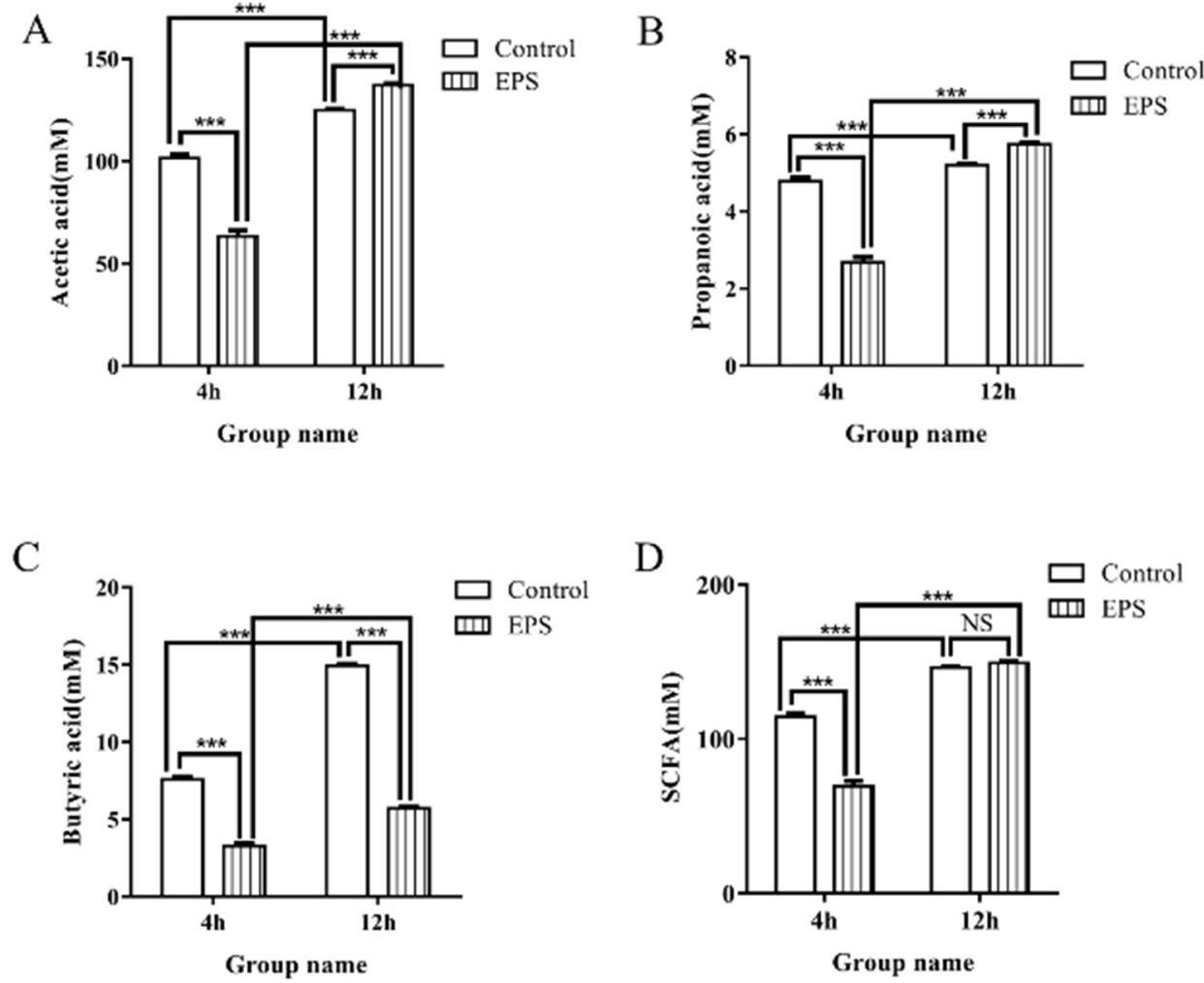

Figure 14. Mean concentrations of acetate (A), propionate (B), butyrate (C), and total short-chain fatty acids (SCFAs) (D) during in vitro fecal fermentation with intervention of the EPS produced by Lpb. plantarum NMGL2 for $4 \mathrm{~h}$ and $12 \mathrm{~h}$. $p$ values $<0.001$ is indicated by ${ }^{* * *}$. NS: no significant.

\section{Conclusions}

In this study, a novel EPS with a low molecular weight $\left(3.03 \times 10^{4} \mathrm{Da}\right)$ produced by Lpb. plantarum NMGL2 isolated from traditional cheese in Inner Mongolia of China was characterized with significant bioactivity by regulating the composition and diversity of fecal microbiota. The EPS produced by Lpb. plantarum NMGL2 had a strong resistance to human digestive juices (saliva, gastric juice, and intestinal juice). It was relatively heat stable with a degrading temperature of about $245^{\circ} \mathrm{C}$. Microstructurally, the EPS appeared to be a highly branched and tangled fibrous network with many hollow microtubules and spherical particles. Monosaccharide analysis indicated that the EPS was composed of $\beta$-galactose $(4.6 \%), \alpha$-glucose $(20.6 \%)$, and $\alpha$-mannose $(74.8 \%)$, and it might contain a tetrasaccharide repeating unit. Analysis of the fecal microbiota regulatory activity of the EPS showed that the EPS affected the composition and diversity of the fecal microbiota by increasing the relative abundance of beneficial bacteria, e.g., Firmicutes, and inhibiting harmful bacteria, e.g., Proteobacteria. The EPS also affected the fecal SCFAs composition during in vitro fecal fermentation, which in turn could regulate the fecal microbiota. Therefore, the low-molecular-weight EPS produced by Lpb. plantarum NMGL2 possessed significant bioactivity, which could be explored for potential applications in the food and health care industry. 


\begin{abstract}
Author Contributions: M.Y. contributed to the study design, carried out the experimental work, performed statistical evaluation, drafted the article. M.Z. interpreted data. T.L. contributed to the conception of the study and experimental design. Z.Y. revised the article. All authors have read and agreed to the published version of the manuscript.
\end{abstract}

Funding: This work was supported by the National Natural Science Foundation of China (project no. 31871823), The National Centre for Research and Development (Narodowe Centrum Badań i Rozwoju) (no. 2017YFE0131800).

Institutional Review Board Statement: Not applicable.

Informed Consent Statement: Not applicable.

Data Availability Statement: Not applicable.

Conflicts of Interest: The authors declare no conflict of interest.

\title{
References
}

1. Lee, I.C.; Caggianiello, G.; van Swam, I.I.; Taverne, N.; Meijerink, M.; Bron, P.A.; Spano, G.; Kleerebezem, M. Strain-Specific Features of Extracellular Polysaccharides and Their Impact on Lactobacillus plantarum-Host Interactions. Appl. Environ. Microbiol. 2016, 82, 3959-3970. [CrossRef] [PubMed]

2. Jiang, Y.Y.; Yang, Z.N. A functional and genetic overview of exopolysaccharides produced by Lactobacillus plantarum. J. Funct. Foods 2018, 47, 229-240. [CrossRef]

3. Zhang, L.; Liu, C.; Li, D.; Zhao, Y.; Zhang, X.; Zeng, X.; Yang, Z.; Li, S. Antioxidant activity of an exopolysaccharide isolated from Lactobacillus plantarum C88. Int. J. Biol. Macromol. 2013, 54, 270-275. [CrossRef] [PubMed]

4. Sun, M.; Liu, W.; Song, Y.; Tuo, Y.; Mu, G.; Ma, F. The Effects of Lactobacillus plantarum-12 Crude Exopolysaccharides on the Cell Proliferation and Apoptosis of Human Colon Cancer (HT-29) Cells. Probiotics Antimicrob. Proteins 2021, 13, 413-421. [CrossRef] [PubMed]

5. Mizuno, H.; Tomotsune, K.; Islam, M.A.; Funabashi, R.; Albarracin, L.; Ikeda-Ohtsubo, W.; Aso, H.; Takahashi, H.; Kimura, K.; Villena, J.; et al. Exopolysaccharides From Streptococcus thermophilus ST538 Modulate the Antiviral Innate Immune Response in Porcine Intestinal Epitheliocytes. Front. Microbiol. 2020, 11, 894. [CrossRef] [PubMed]

6. Hassan, A.N.; Ipsen, R.; Janzen, T.; Qvist, K.B. Microstructure and rheology of yogurt made with cultures differing only in their ability to produce exopolysaccharides. J. Dairy Sci. 2003, 86, 1632-1638. [CrossRef]

7. Krembs, C.; Eicken, H.; Junge, K.; Deming, J.W. High concentrations of exopolymeric substances in Arctic winter sea ice: Implications for the polar ocean carbon cycle and cryoprotection of diatoms. Deep Sea Res. Pt. I 2002, 49, 2163-2181. [CrossRef]

8. Shi, Y.; Xiong, Q.; Wang, X.; Li, X.; Yu, C.; Wu, J.; Yi, J.; Zhao, X.; Xu, Y.; Cui, H. Characterization of a novel purified polysaccharide from the flesh of Cipangopaludina chinensis. Carbohydr. Polym. 2016, 136, 875-883. [CrossRef]

9. Zhou, Y.; Cui, Y.; Qu, X. Exopolysaccharides of lactic acid bacteria: Structure, bioactivity and associations: A review. Carbohydr. Polym. 2019, 207, 317-332. [CrossRef] [PubMed]

10. Guillon, F.; Champ, M. Structural and physical properties of dietary fibres, and consequences of processing on human physiology. Food Res. Int. 2000, 33, 233-245. [CrossRef]

11. Wang, Y.; Liu, G.; Liu, R.; Wei, M.; Zhang, J.; Sun, C. EPS364, a Novel Deep-Sea Bacterial Exopolysaccharide, Inhibits Liver Cancer Cell Growth and Adhesion. Mar. Drugs 2021, 19, 171. [CrossRef] [PubMed]

12. Tang, W.; Zhou, J.; Xu, Q.; Dong, M.; Fan, X.; Rui, X.; Zhang, Q.; Chen, X.; Jiang, M.; Wu, J.; et al. In vitro digestion and fermentation of released exopolysaccharides (r-EPS) from Lactobacillus delbrueckii ssp. bulgaricus SRFM-1. Carbohydr. Polym. 2020, 230, 115593. [CrossRef] [PubMed]

13. Looijesteijn, P.J.; Trapet, L.; de Vries, E.; Abee, T.; Hugenholtz, J. Physiological function of exopolysaccharides produced by Lactococcus lactis. Int. J. Food Microbiol. 2001, 64, 71-80. [CrossRef]

14. Vinderola, G.; Perdigon, G.; Duarte, J.; Farnworth, E.; Matar, C. Effects of the oral administration of the exopolysaccharide produced by Lactobacillus kefiranofaciens on the gut mucosal immunity. Cytokine 2006, 36, 254-260. [CrossRef] [PubMed]

15. Hu, J.-L.; Nie, S.-P.; Min, F.-F.; Xie, M.-Y. Artificial simulated saliva, gastric and intestinal digestion of polysaccharide from the seeds of Plantago asiatica L. Carbohydr. Polym. 2013, 92, 1143-1150. [CrossRef] [PubMed]

16. Larsen, N.; Cahu, T.B.; Isay Saad, S.M.; Blennow, A.; Jespersen, L. The effect of pectins on survival of probiotic Lactobacillus spp. in gastrointestinal juices is related to their structure and physical properties. Food Microbiol. 2018, 74, 11-20. [CrossRef] [PubMed]

17. Chen, C.; Zhang, B.; Fu, X.; You, L.-J.; Abbasi, A.M.; Liu, R.H. The digestibility of mulberry fruit polysaccharides and its impact on lipolysis under simulated saliva, gastric and intestinal conditions. Food Hydrocoll. 2016, 58, 171-178. [CrossRef]

18. Mao, Y.H.; Song, A.X.; Li, L.Q.; Siu, K.C.; Yao, Z.P.; Wu, J.Y. Effects of exopolysaccharide fractions with different molecular weights and compositions on fecal microflora during in vitro fermentation. Int. J. Biol. Macromol. 2020, 144, 76-84. [CrossRef]

19. Salazar, N.; Gueimonde, M.; Hernandez-Barranco, A.M.; Ruas-Madiedo, P.; de los Reyes-Gavilan, C.G. Exopolysaccharides produced by intestinal Bifidobacterium strains act as fermentable substrates for human intestinal bacteria. Appl. Environ. Microbiol. 2008, 74, 4737-4745. [CrossRef] 
20. Guo, H.; Xue, S.; Nasir, M.; Lv, J.; Gu, J. Role of Bentonite on the Mobility of Antibiotic Resistance Genes, and Microbial Community in Oxytetracycline and Cadmium Contaminated Soil. Front. Microbiol. 2018, 9, 2722. [CrossRef] [PubMed]

21. Zheng, X.; Qiu, Y.; Zhong, W.; Baxter, S.; Su, M.; Li, Q.; Xie, G.; Ore, B.M.; Qiao, S.; Spencer, M.D.; et al. A targeted metabolomic protocol for short-chain fatty acids and branched-chain amino acids. Metabolomics 2013, 9, 818-827. [CrossRef] [PubMed]

22. Li, C.C.; Li, W.; Chen, X.H.; Feng, M.Q.; Rui, X.; Jiang, M.; Dong, M.S. Microbiological, physicochemical and rheological properties of fermented soymilk produced with exopolysaccharide (EPS) producing lactic acid bacteria strains. LWT Food Sci Technol. 2014, 57, 477-485. [CrossRef]

23. Kavita, K.; Mishra, A.; Jha, B. Extracellular polymeric substances from two biofilm forming Vibrio species: Characterization and applications. Carbohydr. Polym. 2013, 94, 882-888. [CrossRef] [PubMed]

24. Ye, S.; Zhang, M.; Yang, H.; Wang, H.; Xiao, S.; Liu, Y.; Wang, J. Biosorption of Cu2+, Pb2+ and Cr6+ by a novel exopolysaccharide from Arthrobacter ps-5. Carbohydr. Polym. 2014, 101, 50-56.

25. Matsuzaki, C.; Takagaki, C.; Tomabechi, Y.; Forsberg, L.S.; Heiss, C.; Azadi, P.; Matsumoto, K.; Katoh, T.; Hosomi, K.; Kunisawa, J.; et al. Structural characterization of the immunostimulatory exopolysaccharide produced by Leuconostoc mesenteroides strain NTM048. Carbohydr. Res. 2017, 448, 95-102. [CrossRef]

26. Shingel, K.I. Determination of structural peculiarities of dexran, pullulan and $\gamma$-irradiated pullulan by Fourier-transform IR spectroscopy. Carbohydr. Res. 2002, 337, 1445-1451. [CrossRef]

27. Wang, X.; Shao, C.; Liu, L.; Guo, X.; Xu, Y.; Lu, X. Optimization, partial characterization and antioxidant activity of an exopolysaccharide from Lactobacillus plantarum KX041. Int. J. Biol. Macromol. 2017, 103, 1173-1184. [CrossRef]

28. Das, D.; Baruah, R.; Goyal, A. A food additive with prebiotic properties of an alpha-d-glucan from Lactobacillus plantarum DM5 Int. J. Biol. Macromol. 2014, 69, 20-26. [CrossRef]

29. İspirli, H.; Sagdic, O.; Y1lmaz, M.T.; Dertli, E. Physicochemical characterisation of an $\alpha$-glucan from Lactobacillus reuteri E81 as a potential exopolysaccharide suitable for food applications. Process. Biochem. 2019, 79, 91-96. [CrossRef]

30. Miao, M.; Ma, Y.; Huang, C.; Jiang, B.; Cui, S.W.; Zhang, T. Physicochemical properties of a water soluble extracellular homopolysaccharide from Lactobacillus reuteri SK24.003. Carbohydr. Polym. 2015, 131, 377-383. [CrossRef]

31. Ale, E.C.; Perezlindo, M.J.; Pavon, Y.; Peralta, G.H.; Costa, S.; Sabbag, N.; Bergamini, C.; Reinheimer, J.A.; Binetti, A.G Technological, rheological and sensory characterizations of a yogurt containing an exopolysaccharide extract from Lactobacillus fermentum Lf2, a new food additive. Food Res. Int. 2016, 90, 259-267. [CrossRef] [PubMed]

32. Galle, S.; Schwab, C.; Arendt, E.K.; Gaenzle, M.G. Structural and rheological characterisation of heteropolysaccharides produced by lactic acid bacteria in wheat and sorghum sourdough. Food Microbiol. 2011, 28, 547-553. [CrossRef] [PubMed]

33. Mathivanan, K.; Chandirika, J.U.; Vinothkanna, A.; Govindarajan, R.K.; Meng, D.L.; Yin, H.Q. Characterization and Biotechnological Functional Activities of Exopolysaccharides Produced by Lysinibacillus fusiformis KMNTT-10. J. Polym. Environ. 2021, 29, 1742-1751. [CrossRef]

34. Ikeda, S.; Murayama, D.; Tsurumaki, A.; Sato, S.; Urashima, T.; Fukuda, K. Rheological characteristics and supramolecular structure of the exopolysaccharide produced by Lactobacillus fermentum MTCC 25067. Carbohydr. Polym. 2019, 218, 226-233. [CrossRef] [PubMed]

35. Chen, L.; Wang, Z.; Zhang, B.; Ge, M.; Ng, H.; Niu, Y.; Liu, L. Production, structure and morphology of exopolysaccharides yielded by submerged fermentation of Antrodia cinnamomea. Carbohydr. Polym. 2019, 205, 271-278. [CrossRef] [PubMed]

36. Jiang, B.; Tian, L.; Huang, X.; Liu, Z.; Jia, K.; Wei, H.; Tao, X. Characterization and antitumor activity of novel exopolysaccharide APS of Lactobacillus plantarum WLPL09 from human breast milk. Int. J. Biol. Macromol. 2020, 163, 985-995. [CrossRef]

37. Wang, Z.; Liu, X.; Bao, Y.; Wang, X.; Zhai, J.; Zhan, X.; Zhang, H. Characterization and anti-inflammation of a polysaccharide produced by Chaetomium globosum CGMCC 6882 on LPS-induced RAW 264.7 cells. Carbohydr. Polym. 2021, 251, 117129. [CrossRef]

38. Frank, D.N.; Robertson, C.E.; Hamm, C.M.; Kpadeh, Z.; Zhang, T.; Chen, H.; Zhu, W.; Sartor, R.B.; Boedeker, E.C.; Harpaz, N.; et al. Disease phenotype and genotype are associated with shifts in intestinal-associated microbiota in inflammatory bowel diseases. Inflamm. Bowel Dis. 2011, 17, 179-184. [CrossRef]

39. Mukhopadhya, I.; Hansen, R.; El-Omar, E.M.; Hold, G.L. IBD—What role do Proteobacteria play? Nat. Rev. Gastroenterol. Hepatol. 2012, 9, 219-230. [CrossRef]

40. Yan, S.; Yang, B.; Zhao, J.; Zhao, J.; Stanton, C.; Ross, R.P.; Zhang, H.; Chen, W. A ropy exopolysaccharide producing strain Bifidobacterium longum subsp. longum YS108R alleviates DSS-induced colitis by maintenance of the mucosal barrier and gut microbiota modulation. Food Funct. 2019, 10, 1595-1608. [CrossRef]

41. Wu, F.; Guo, X.; Zhang, J.; Zhang, M.; Ou, Z.; Peng, Y. Phascolarctobacterium faecium abundant colonization in human gastrointestinal tract. Exp. Ther. Med. 2017, 14, 3122-3126. [CrossRef] [PubMed]

42. Sokol, H.; Pigneur, B.; Watterlot, L.; Lakhdari, O.; Bermudez-Humaran, L.G.; Gratadoux, J.-J.; Blugeon, S.; Bridonneau, C.; Furet, J.-P.; Corthier, G.; et al. Faecalibacterium prausnitzii is an anti-inflammatory commensal bacterium identified by gut microbiota analysis of Crohn disease patients. Proc. Natl. Acad. Sci. USA 2008, 105, 16731-16736. [CrossRef] [PubMed]

43. Reichardt, N.; Duncan, S.H.; Young, P.; Belenguer, A.; Leitch, C.M.; Scott, K.P.; Flint, H.J.; Louis, P. Erratum: Phylogenetic distribution of three pathways for propionate production within the human gut microbiota. ISME J. 2014, 8, 1323-1335. [CrossRef] [PubMed]

44. Louis, P.; Flint, H.J. Formation of propionate and butyrate by the human colonic microbiota. Environ. Microbiol. 2017, 19, 29-41. [CrossRef] [PubMed] 
45. Guarner, F.; Khan, A.G.; Garisch, J.; Eliakim, R.; Szajewska, H.J.S.A.G.R. World Gastroenterology Organisation practice guideline: Probiotics and prebiotics. Arab J. Gastroenterol. 2009, 10, 33-42.

46. Li, A.L.; Ni, W.W.; Li, Y.; Zhang, X.; Yang, J.J.; Ma, X.Y.; Jia, X.D.; Li, C.; Liu, L.B. Effect of 2'-fucosyllactose supplementation on intestinal flora in mice with intestinal inflammatory diseases. Int. Dairy J. 2020, 110, 104797. [CrossRef]

47. Peng, M.; Biswas, D. Short chain and polyunsaturated fatty acids in host gut health and foodborne bacterial pathogen inhibition. Crit. Rev. Food Sci. 2017, 57, 3987-4002. [CrossRef] [PubMed]

48. Holscher, H.D.; Bauer, L.L.; Gourineni, V.; Pelkman, C.L.; Fahey, G.C., Jr.; Swanson, K.S. Agave Inulin Supplementation Affects the Fecal Microbiota of Healthy Adults Participating in a Randomized, Double-Blind, Placebo-Controlled, Crossover Trial. J. Nutr. 2015, 145, 2025-2032. [CrossRef] [PubMed]

49. Li, Z.; Yi, C.X.; Katiraei, S.; Kooijman, S.; Zhou, E.; Chung, C.K.; Gao, Y.; van den Heuvel, J.K.; Meijer, O.C.; Berbee, J.F.P.; et al. Butyrate reduces appetite and activates brown adipose tissue via the gut-brain neural circuit. Gut 2018, 67, 1269-1279. [CrossRef] 